\title{
A QUASICONFORMAL GROUP NOT ISOMORPHIC TO A MÖBIUS GROUP
}

\author{
PEKKA TUKIA
}

1. Introduction. We describe here an open $(n-1)$-cell in $R^{n}, n \geqq 3$, which can be used to construct counterexamples to several natural conjectures in quasiconformal mapping theory. The most important of these provides a negative answer to a question raised by F. W. Gehring and B. P. Palka in [6, p. 197]. Their question, slightly simplified, is whether every uniformly quasiconformal group on $R^{n}$ is of the form $f G f^{-1}$ where $f$ is a quasiconformal homeomorphism of $R^{n}$ and $G$ is a group of Möbius transformations on $R^{n}$. Our Example 1 shows that this is not always the case. We present our examples in Section 5.

The $(n-1)$-cell $S$ to be exhibited is of the form $J \times R^{n-2}$ where $J \subset R^{2}$ is a locally non-rectifiable arc such that $J \cup\{\infty\}$ is a quasicircle, i.e. $J \cup\{\infty\}=g\left(S^{1}\right)$ for some quasiconformal homeomorphism of $\bar{R}^{2}$. Thus the pathology of $S$ derives from the fact that it is highly anisotropic. In some directions it is rectifiable, in other directions it is not. This precludes the possibility that $S$ is the image of an $(n-1)$-plane under a quasiconformal self-mapping of $R^{n}$ (cf. Theorem 5).

Nevertheless, there is a group $G_{0}$ of homeomorphisms of $R^{n}$ which acts transitively on $S$ and which is uniformly Lipschitz (and hence uniformly quasiconformal). One can show that, if $h$ is a homeomorphism of $R^{n}$ such that $h G_{0} h^{-1}$ is a group of Möbius transformations, then $h(S)$ is necessarily an $(n-1)$-plane. In view of the preceding remark, such a map cannot be quasiconfcrmal, although there are nonquasiconformal $h$ for which $h G_{0} h^{-1}$ is a Möbius group. Furthermore, there is a uniformly quasiconformal group $G_{1} \supset G_{0}$ acting transitively on the complementary domains of $S$ such that $G_{1}$ is not isomorphic as a topological group to any group of Möbius transformations on some $\bar{R}^{m}, m>0$.

In contrast, every uniformly quasiconformal group on $R^{2}$ is a quasiconformal conjugate of some Möbius group [8, 10]. This is due to the fact that, although for each countable quasiconformal group $G$ on $\bar{R}^{n}$ one can find a $G$-invariant conformal structure on $\bar{R}^{n}$, it is only for $\bar{R}^{2}$ that such a structure can always be realized as a pull-back of the standard conformal structure of $\bar{R}^{2}$.

I wish to acknowledge that it was S. Rickman who first discovered that $S \cup\{\infty\}$ is not a quasiconformal sphere (for $n=3$ ). The proof of this fact which we present here (slightly generalized) is due to J. Väisälä. I wish to thank them for giving 
me this example and this proof. I am also indebted for the referee for his detailed report.

Throughout this paper $n \geqq 3$ is a fixed integer.

2. Some definitions. Let $X$ and $Y$ be metric spaces. Then an embedding $f: X \rightarrow Y$ is said to be bilipschitz (or L-bilipschitz) if, for some $L \geqq 1$,

$$
|x-y| / L \leqq|f(x)-f(y)| \leqq L|x-y|
$$

for all $x, y \in X$, where the notation $|a-b|$ is used for the distance between points $a$ and $b$. It is said to be quasisymmetric (or H-quasisymmetric) if there is $H \geqq 1$ such that

$$
|f(a)-f(x)| \leqq H|f(b)-f(x)|
$$

for all $a, b, x \in X$ satisfying $|a-x| \leqq|b-x|$. (Cf. [12], where the terminology weakly quasisymmetric was used for an embedding satisfying (2).)

Let $U \subset R^{n}, U \neq R^{n}$, be open and connected. Then the quasihyperbolic metric $q$ of $U$ is defined by the metric density $\varrho(x)=1 / d(x, \partial U)$ (where $d(x, \partial U)$ is the euclidean distance of $x$ from the boundary $\partial U$ of $U$ ), cf. [6, Section 2]. If $U=H^{n}=$ $\left\{\left(x_{1}, \ldots, x_{n}\right) \in R^{n}: x_{n}>0\right\}$ is the $n$-dimensional hyperbolic space, then the quasihyperbolic metric of $H^{n}$ is just the hyperbo'ic metric $p$ of $H^{n}$. If $U \subset R^{2}$ and $\partial U \cup\{\infty\}$ consists of more than three points, then $U$ has a unique Riemannian structure of constant curvature -1 such that the conformal structure of $U$ in this metric is the same as the conformal structure as a subset of $R^{2}=$ the complex plane. The metric of $U$ defined by this structure is the hyperbolic metric $p$ of $U$. If $U$ is simply connected there is the following relation between the metrics $q$ and and $p$ of $U$

$$
q(x, y) / 2 \leqq p(x, y) \leqq 2 q(x, y)
$$

for all $x, y \in U$, cf. Ahlfors [1, p. 79].

If $X$ is a metric space and $G$ is a group of homeomorphisms of $X$, we say that $X$ is a Lipschitz group on $X$ if there is $L \geqq 1$ such that every $g \in G$ is $L$-bilipschitz. If $U \subset \bar{R}^{n}=R^{n} \cup\{\infty\}$ is open, if $G$ is a group of homeomorphisms of $U$ and if there is $K \geqq 1$ such that every $g \in G$ is $K$-quasiconformal, $G$ is a quasiconformal group ( $\subset n U$ ). If every $g \in G$ is a Möbius transformation, $G$ is a Möbius group. These groups are also topological groups in the topology of uniform convergence on compact subsets.

We say that a $k$-manifold $M \subset R^{n}$ is quasiconformally locally flat if for every $x \in M$ there is a neighbourhood $U$ of $x$ in $R^{n}$ and a quasiconformal embedding $f: U \rightarrow R^{n}$ such that $f(M) \subset R^{k}$. A set $X \subset \bar{R}^{n}$ is a $k$-quasisphere if $X=f\left(S^{k}\right)$ for some quasiconformal $f: \bar{R}^{n} \rightarrow \bar{R}^{n}$.

We let $I=[0,1]$ be the unit interval. In a metric space $X, B(x, r)$ is the closed ball with center $x$ and radius $r$. If $X=R^{k}$, we also write $B^{k}(x, r)$ for $B(x, r)$. The unit sphere of $R^{k}$ is $S^{k-1}=\partial B^{k}(x, 1)$ where $\partial A$ is the boundary of a set $A$. The euclidean distances of a point $x$ or a set $B$ from $A$ are $d(x, A)$ and $d(B, A)$; similar notations are used for other metrics. 

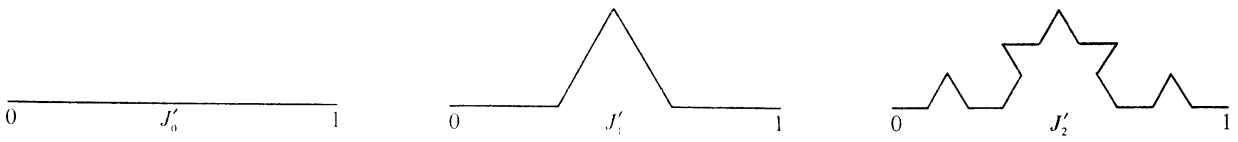

Figure 1

3. Construction of the groups. We first define the arc $J$ mentioned in the Introduction. Consider the arcs $J_{0}^{\prime}, J_{1}^{\prime}, \ldots$ as in Figure 1. These converge to a wellknown non-rectifiable quasiconformal arc $J^{\prime}$. Observe that $J^{\prime} / 3$ is a subarc of $J^{\prime}$ and likewise $J^{\prime}$ is a subarc of $3 J^{\prime}$. Thus if we set

$$
J=\bigcup_{i \geqq 0} 3^{i}\left(J^{\prime} \cup\left(-J^{\prime}\right)\right)
$$

we obtain an (open) $\operatorname{arc} J$ with the property that $J \cup\{\infty\}$ is a quasicircle.

There is a natural map $f: R \rightarrow J$, defined as follows. Each arc $J_{k}^{\prime}$ consists of $4^{k}$ segments $J_{k i}^{\prime}, i=1, \ldots, 4^{k}$, of equal length. Let these be in order on $J_{k}^{\prime}$ with $0 \in J_{k 1}^{\prime}$. Let $I_{k i}=\left[(i-1) 4^{-k}, i 4^{-k}\right]$ and let $f_{k}^{\prime}: I \rightarrow J_{k}^{\prime}$ be the map such that $f_{k}^{\prime}\left(I_{k i}\right)=$ $J_{k i}^{\prime}$ and that $f_{k}^{\prime} \mid I_{k i}$ is affine. Then the maps $f_{k}$ converge to a map $f^{\prime}: I \rightarrow J^{\prime}$ such that $f^{\prime}\left(4^{i} x\right)=3^{i} f^{\prime}(x)$ if $i \geqq 0$ and $0 \leqq x \leqq 4^{i} x \leqq 1$. We define $f$ by

$$
f\left( \pm 4^{i} x\right)= \pm 3^{i} f^{\prime}(x)
$$

if $x \in[0,1]$ and $i \geqq 0$. Then $f$ is a homeomorphism $R \rightarrow J$ and there is $M \geqq 1$ such that if $\alpha=\log 3 / \log 4(1 / \alpha=$ the Hausdorff dimension of $J)$,

$$
|x-y|^{x} / M \leqq|f(x)-f(y)| \leqq M|x-y|^{x}
$$

for all $x, y \in R$. To see the validity of (4), observe first that if $x$ and $y$ are the endpoints of some interval $I_{k i}=\left[(i-1) 4^{-k}, i 4^{-k}\right], i, k \in Z$, then (4) is true with $M=1$. If $x, y$ are arbitrary, we can compare $|f(x)-f(y)|$ to some distances $\left|f\left(x^{\prime}\right)-f\left(y^{\prime}\right)\right|$ where $x^{\prime}$ and $y^{\prime}$ are the endpoints of a suitable interval $I_{k i}$. In this manner we find $M>1$ satisfying (4) for all $x, y \in R$.

It is a consequence of (4) that $f$ is quasisymmetric. It satisfies (2) in the euclidean metric with $H=M^{2}$. Then, by [11], we can extend $f$ to a quasiconformal homeomorphism $F^{\prime}$ of $R^{2}$. It follows that for some $M^{\prime} \geqq 1$

$$
|v|^{x} / M^{\prime} \leqq d\left(F^{\prime}(u, v), J\right) \leqq M^{\prime}|v|^{\alpha}
$$

for all $u, v \in R$. This can be seen with the aid of (4), since quasiconformal maps of $R^{2}$ are quasisymmetric (by $[14,2.4]$ or by a normal family argument). Indeed, if $F^{\prime}$ is $H$-quasisymmetric, then $d\left(F^{\prime}(u, v), J\right) \leqq\left|F^{\prime}(u, v)-f(u)\right| \leqq H|f(u+v)-f(\mu)| \leqq$ $H M|v|^{\alpha}$. On the other hand, let $y=f(x) \in J$ be a point such that $d\left(F^{\prime}(u, v), J\right)=$ $\left|F^{\prime}(u, v)-y\right|$. Then $|(u, v)-x| \geqq|(u, v)-u|$ and thus $d\left(F^{\prime}(u, v), J\right)=\left|F^{\prime}(u, v)-y\right| \geqq$ $\left|F^{\prime}(u, v)-f(u)\right| / H \geqq|f(u+v)-f(u)| / H^{2} \geqq|v|^{\alpha} / M H^{2}$. Thus (5) is true with $M^{\prime}=M H^{2}$. 
We will require that $F^{\prime}$ be chosen in such a way that the map $F^{\prime}$ is bilipschitz in the quasihyperbolic metric when restricted to a component $C$ of $R^{2} \backslash R$. Thus we require that

$$
q(x, y) / L \leqq q\left(F^{\prime}(x), F^{\prime}(y)\right) \leqq L q(x, y)
$$

for some $L \geqq 1$ whenever $x, y \in C$, where the notation $q$ is used for the quasihyperholic metric in both $C$ and $F^{\prime}(C)$. This follows since in [11] $F^{\prime}$ was constructed as a composition $F^{\prime} \mid C=g h$ where $g: C \rightarrow F^{\prime}(C)$ is conformal and $h: C \rightarrow C$ is the Beurling-Ahlfors extension of a quasisymmetric map of the real line. Now by $[1$, p. 73], $h$ is bilipschitz in the hyperbolic metric $p=q$ of $C$ and the map $g$ is an isometry in the hyperbolic metrics of $C$ and $F^{\prime}(C)$. Since the metrics $q$ and $p$ of $F^{\prime}(C)$ are bilipschitz equivalent by (3), (6) follows.

Let now $G_{0}^{\prime}$ be the group consisting of all translations of $R^{2}$ parallel to the real axis, that is, every $g \in G_{0}^{\prime}$ is of the form $x \mapsto x+a$ for some $a \in R$. Let $G_{1}^{\prime}$ be the group generated by $G_{0}^{\prime}$ and by the maps $g_{\lambda}^{\prime}: R^{2} \rightarrow R^{2}, g_{\lambda}^{\prime}(x)=\lambda x$, for $\lambda>0$. Let $G_{0}^{\prime \prime}=F^{\prime} G_{0}^{\prime} F^{\prime-1}$ and $G_{1}^{\prime \prime}=F^{\prime} G_{1}^{\prime} F^{\prime-1}$. Then we have

Lemma 1. (a) The group $G_{0}^{\prime \prime}$ is a Lipschitz group of $R^{2}$ in the euclidean metric.

(b) The group $G_{1}^{\prime \prime}$ is a quasiconformal group of $R^{2}$ such that if the action of $G_{1}^{\prime \prime}$ is restricted to a component $C^{\prime}$ of $R^{2} \backslash J$, then $G_{1}^{\prime \prime}$ is a Lipschitz group in the quasihyperbolic metric of $C^{\prime}$.

Proof of (a). We first show that there is $L_{0} \geqq 1$ such that if $g \in G_{0}^{\prime \prime}$, if $x \in R^{2} \backslash J$ and if $g$ is differentiable at $x$, then the differential $D g(x)$ of $g$ at $x$ satisfies

$$
1 / L_{0} \leqq l(D g(x)) \leqq|D g(x)| \leqq L_{0}
$$

where $l(A)=\inf _{|u|=1}|A(u)|,|A|=\sup _{|u|=1}|A(u)|$ for a linear map $A$ of $R^{2}$. Let $C_{0}$ be the component of $R^{2} \backslash R$ such that $x \in F^{\prime}\left(C_{0}\right)$. Now $g=F^{\prime} g^{\prime} F^{\prime-1}$ for some $g^{\prime} \in G_{0}^{\prime}$. Here $g^{\prime}$ is an isometry in the quasihyperbolic metric $q=p$ of $C_{0}$. Then (6) implies that $g$ is $L^{2}$-bilipschitz in the quasihyperbolic metric $q$ of $F^{\prime}\left(C_{0}\right)$. Observe that $x$ and $g(x)$ are in a set $A_{v}=F^{\prime}(R \times\{v\})$ for some $v \neq 0$. Thus, by (5), $1 / M^{\prime 2} \leqq$ $d(g(x), J) / d(x, J) \leqq M^{\prime 2}$. It follows that (7) is true with $L_{0}=L^{2} M^{\prime 2}$.

Now the map $g$ is quasiconformal. Thus $g$ and $g^{-1}$ are ACL and a.e. differentiable. Since we now know that (7) is true a.e. outside $J$ and hence a.e. in $R^{2}, g$ must be $L_{0}$-bilipschitz.

Case (b) is obvious by (6) and by the quasiconformality of $F^{\prime}$.

We now define maps $\bar{h}_{a}$ and $\bar{g}_{\lambda}$ of $R^{n}$ for $a \in R^{n}$ and $\lambda>0$ by

$$
\begin{aligned}
\bar{h}_{a}(z) & =z+a, \\
\bar{g}_{\lambda}(x, y) & =\left(\lambda x, \lambda^{\alpha} y\right)
\end{aligned}
$$

if $z \in R^{n},(x, y) \in R^{2} \times R^{n-2}$, with $\alpha=\log 3 / \log 4$ as in (4). Next we define the groups 
$\bar{G}_{0}$ and $\bar{G}_{1}$ of affine maps of $R^{n}$ by

$$
\begin{aligned}
& \bar{G}_{0}=\left\{\bar{h}_{a}: a=\left(a_{1}, 0, a_{3}, \ldots, a_{n}\right), a_{i} \in R\right\} \text { and } \\
& \bar{G}_{1}=\text { the group generated by } \bar{G}_{0} \text { and the maps } \bar{g}_{\lambda}, \lambda>0 .
\end{aligned}
$$

Then every $g \in \bar{G}_{1}$ is of the form $g(x, y)=\left(\lambda x+a, \lambda^{\alpha} y+b\right),(x, y) \in R^{2} \times R^{n-2}$, for some $\lambda>0, a=\left(a^{\prime}, 0\right) \in R^{2}$ and $b \in R^{n-2}$.

Let then $F=F^{\prime} \times \mathrm{id}: R^{n} \rightarrow R^{n}$ and set

$$
\begin{array}{ll}
h_{a}=F \bar{h}_{a} F^{-1}, & g_{\lambda}=F \bar{g}_{\lambda} F^{-1}, \\
G_{0}=F \bar{G}_{0} F^{-1}, & G_{1}=F \bar{G}_{1} F^{-1} .
\end{array}
$$

Finally we observe the following commutativity relations between the maps $h_{a}$ and $g_{\lambda}$ :

$$
\begin{gathered}
h_{\lambda a}=g_{\lambda} h_{a} g_{\lambda}^{-1} \text { for } a=\left(a_{1}, 0, \ldots, 0\right), \text { but } \\
h_{\lambda^{\alpha} b}=g_{\lambda} h_{b} g_{\lambda}^{-1} \text { for } \quad b=\left(0,0, b_{3}, \ldots, b_{n}\right)
\end{gathered}
$$

which are immediate consequences of the definitions. We have

Theorem 2. (a) The group $G_{0}$ is a Lipschitz group of $R^{n}$ in the euclidean metric acting transitively on $S=J \times R^{n-2}$.

(b) The group $G_{1}$ is a quasiconformal group of $R^{n}$ acting transitively on $S$ and on each component of $R^{n} \backslash S$. If the action of $G_{1}$ is restricted to a component $C$ of $R^{n} \backslash S$, then $G_{1}$ is a Lipschitz group of $C$ in the quasihyperbolic metric.

Proof. Case (a) is obvious by Lemma 1 (a) and by the definition of $G_{0}$.

We then prove (b). Let $C$ be as indicated. Obviously $G_{1}$ acts transitively on $S$ and $C$. We show that $G_{1}$ is a Lipschitz group of $C$ in the quasihyperbolic metric. By (9), every $g \in G_{1}$ can be represented in the form $g=h_{a} g_{\lambda}$ for some $\lambda>0$ and $a=\left(a_{1}, 0, a_{3}, \ldots, a_{n}\right) \in R^{n}$. Thus it suffices to show that the maps $h_{a} \mid C$ and $g_{\lambda} \mid C$ are uniformly bilipschitz in the quasihyperbolic metric. For the maps $h_{a} \mid C$ this is clear by case (a) since, by (5), $d(x, S) / M^{\prime} \leqq d\left(h_{a}(x), S\right) \leqq M^{\prime} d(x, S)$ for all $x \in C$ and $a=\left(a_{1}, 0, a_{3}, \ldots, a_{n}\right) \in R^{n}$.

We consider next the maps $g_{\lambda}$. Let $x \in C, x=\left(x^{\prime}, y^{\prime}\right) \in R^{2} \times R^{n-2}$. Then $g_{\lambda}(x)=$ $\left(g_{\lambda}^{\prime \prime}\left(x^{\prime}\right), \lambda^{\alpha} y^{\prime}\right)$ where $g_{\lambda}^{\prime \prime}\left(x^{\prime}\right)=F^{\prime}\left(\lambda F^{\prime-1}\left(x^{\prime}\right)\right)$. Let $d_{1}=d(x, S)=d\left(x^{\prime}, J\right)$ and $d_{2}=$ $d\left(g_{\lambda}(x), S\right)=d\left(g_{\lambda}^{\prime \prime}\left(x^{\prime}\right), J\right)$. Let $x^{\prime}=F^{\prime}(u, v), u, v \in R$. Then, by (5), $|v|^{\alpha} / M^{\prime} \leqq d_{1} \leqq$ $|v|^{\alpha} M^{\prime}$ and $|\lambda v|^{\alpha} / M^{\prime} \leqq d_{2} \leqq|\lambda v|^{\alpha} M^{\prime}$. Thus

$$
\lambda^{\alpha} d_{1} / M^{\prime 2} \leqq d_{2} \leqq \lambda^{\alpha} d_{1} M^{\prime 2} .
$$

Now $C=C^{\prime} \times R^{n-2}$ for a component $C^{\prime}$ of $R^{2 \backslash} \backslash$. By Lemma 1 (b), $g_{\lambda}^{\prime \prime}$ is $L$-bilipschitz in the quasihyperbolic metric of $C^{\prime}$ for some $L$ not depending on $\lambda$. Thus, in view of (10), if $g_{\lambda}^{\prime \prime}$ is differentiable at $x$,

$$
\lambda^{\alpha} / L M^{\prime 2} \leqq l\left(D g_{\lambda}^{\prime \prime}\left(x^{\prime}\right)\right) \leqq\left|D g_{\lambda}^{\prime \prime}\left(x^{\prime}\right)\right| \leqq \lambda^{\alpha} L M^{\prime 2} .
$$


Let $h_{\lambda}^{\prime \prime}: R^{n-2} \rightarrow R^{n-2}$ be the map $y \mapsto \lambda^{\alpha} y$. Then $l\left(D h_{\lambda}^{\prime \prime}(y)\right)=\left|D h_{\lambda}^{\prime \prime}(y)\right|=\lambda^{\alpha}$. Since $g_{\lambda}\left(x^{\prime}, y^{\prime}\right)=\left(g_{\lambda}^{\prime \prime}\left(x^{\prime}\right), h_{\lambda}^{\prime \prime}\left(y^{\prime}\right)\right)$, this fact and (11) imply

$$
\lambda^{\alpha} / L M^{\prime 2} \leqq l\left(D g_{\lambda}(x)\right) \leqq\left|D g_{\lambda}(x)\right| \leqq \lambda^{\alpha} L M^{\prime 2} .
$$

Now $g_{\lambda} \mid C$ is in any case locally bilipschitz by (8) and Lemma 1 (b). Then (12) and (10) imply that $g_{\lambda} \mid C$ is $L^{\prime}$-bilipschitz in the quasihyperbolic metric for some $L^{\prime}$ not depending on $\lambda$.

It follows that $G_{1}$ is also a quasiconformal group of $C$ and hence of $R^{n} \backslash S$, since $C$ was an arbitrary component of $R^{n} \backslash S$. Now the $n$-measure of $S$ is zero and it is easy to verify that every $g \in G_{1}$ is ACL. Then the analytic definition of quasiconformality $([15,34.6])$ implies that $G_{1}$ is a quasiconformal group of $R^{n}$.

4. Products of arcs. Now we prove that $S$ is not quasiconformally locally flat. In the following two lemmas we have generalized an argument originally due to J. Väisälä.

We fix now a metric arc $A$, i.e. $A$ is a metric space homeomorphic to a closed interval. The distance of two points $x, y \in A$ is denoted by $|x-y|$.

Lem ma 3. Let $l(A) \in(0, \infty]$ be the length of $A$. Let $M, \delta>0$. Then there is a subdivision of $A$ into subarcs $A_{1}, \ldots, A_{r}$ by successive points $x_{0}, \ldots, x_{r} \in A$ such that, setting $\lambda_{i}=\left|x_{i}-x_{i-1}\right|$, we have

(a) $\lambda_{1}+\ldots+\lambda_{r} \geqq \min (M, l(A)-1 / M)$,

(b) $1 / 2 \leqq \lambda_{i} / \lambda_{j} \leqq 2, \quad i, j \leqq r$, and

(c) $\lambda_{i} \leqq \delta, \quad i \leqq r$.

Proof. In any case there is a subdivision $A_{1}, \ldots, A_{r}$ for which (a) is true. Set $\alpha=(1 / 2) \min \left(\delta, \lambda_{1}, \ldots, \lambda_{r}\right)$. Then $\lambda_{i} \geqq 2 \alpha$. We subdivide each $A_{i}$ as follows. Let $y_{1}$ be the last point on $A_{i}$ (from $x_{i-1}$ ) which is on $B\left(x_{i-1}, \alpha\right)$. If $\left|y_{1}-x_{i}\right|<2 \alpha$ we stop. Otherwise, let $y_{2}$ be the last point of $A_{i}$ on $B\left(y_{1}, \alpha\right)$. Continuing in this manner we get a new subdivision $\left(x_{i-1}=y_{0}, y_{1}, \ldots, y_{k}=x_{i}\right)$ of $A_{i}$ such that each distance $\left|y_{j}-y_{j-1}\right|=\alpha$ except possibly the last one for which $\alpha \leqq\left|y_{k}-y_{k-1}\right|<2 \alpha$. The new subdivision again satisfies (a), as well as (b) and (c).

Lemma 4. Let $A$ be a non-rectifiable metric arc and let $k \geqq 1$. Then $A \times I^{k}=$ $A \times[0,1]^{k}$ cannot be embedded into $R^{k+1}$ by a quasisymmetric map when the metric of $A \times I^{k}$ is given by

$$
\left|(x, y)-\left(x^{\prime}, y^{\prime}\right)\right|=\left(\left|x-x^{\prime}\right|^{2}+\left|y-y^{\prime}\right|^{2}\right)^{1 / 2} .
$$

Proof. Let $h: A \times I^{k} \rightarrow R^{k+1}$ be $H$-quasisymmetric. We show that this leads to a contradiction. Set $I_{a}=\{a\} \times I^{k}$ for $a \in A$. Then every $h \mid I_{a}$ is also $H$-quasisymmetric and, since $I_{a}$ is isometric to $I^{k}$, there is by $[12,2.15$ and 2.1] a map 
$\eta^{\prime}:(0, \infty) \rightarrow(0, \infty)$ such that

$$
|h(x)-h(z)| \geqq \eta^{\prime}(\varrho)|h(y)-h(z)|
$$

whenever $x, y, z \in I_{a}$ for some $a \in A$ and $|x-z| \geqq \varrho|y-z|$.

Choose now $M>0$ and let $\delta=1 / 2$. Choose the subdivision $A_{1}, \ldots, A_{r}$ of $A$ by points $x_{0}, \ldots, x_{r}$ as in Lemma 2 . Setting $\lambda=\max \lambda_{i}$, we have $\lambda / 2 \leqq \lambda_{i} \leqq \lambda \leqq 1 / 2$ for all $i$. Next we subdivide $I$ by points $y_{0}=0, y_{1}, \ldots, y_{s}=1$ into intervals $B_{j}=$ $\left[y_{j-1}, y_{j}\right]$ of equal length $\mu=1 / s$ such that $\lambda \leqq \mu \leqq 2 \lambda \leqq 1$. If $v=\left(j_{1}, \ldots, j_{k}\right), j_{i} \leqq s$, we sêt $B_{v}=B_{j_{1}} \times \ldots \times B_{j_{k}}$. Let $z_{j}=\left(y_{j-1}+y_{j}\right) / 2$ and $z_{v}=\left(z_{j_{1}}, \ldots, z_{j_{k}}\right)\left(z_{v}\right.$ is the center of $B_{v}$ ).

Set now $Q_{i v}=$ int $A_{i} \times B_{v}$. Choose $z_{i}^{\prime} \in A_{i}$ such that $\left|z_{i}^{\prime}-x_{i}\right|=\lambda_{i}|2=| x_{i}-x_{i-1} \mid / 2$ and set $z_{i v}=\left(z_{i}^{\prime}, z_{v}\right)$. Then, if $z \in \partial Q_{i v},\left|z-z_{i v}\right| \geqq \min \left(\mu / 2, \lambda_{i} / 2\right)=\lambda_{i} / 2 \geqq \lambda / 4$. Let $a_{i v}=\left(x_{i}, z_{v}\right)$. Then $\left|a_{i v}-z_{i v}\right|=\left|x_{i}-z_{i}^{\prime}\right|=\lambda_{i} / 2$ and thus $\left|z-z_{i v}\right| \geqq\left|a_{i v}-z_{i v}\right|$ for all $z \in \partial Q_{i v}$. Hence $\left|h(z)-h\left(z_{i v}\right)\right| \geqq\left|h\left(z_{i v}\right)-h\left(a_{i v}\right)\right| / H$. Choose now $y_{i v}^{\prime} \in\left[z_{j_{1}}, y_{j_{1}}\right]$ such that $\quad\left|y_{i v}^{\prime}-z_{j_{1}}\right|=\lambda_{i} / 2=\left|z_{i v}-a_{i v}\right| \quad$ and let $b_{i v}^{\prime}=\left(x_{i}, y_{i v}^{\prime}, y_{j_{2}}, \ldots, y_{j_{k}}\right), \quad b_{i v}=$ $\left(x_{i}, y_{j_{1}}, y_{j_{2}}, \ldots, y_{j_{k}}\right)$ and $b_{i v}^{\prime \prime}=\left(x_{i}, y_{j_{1}-1}, y_{j_{2}}, \ldots, y_{j_{k}}\right)$. Then

$$
\left|h(z)-h\left(z_{i v}\right)\right| \geqq\left|h\left(z_{i v}\right)-h\left(a_{i v}\right)\right| / H \geqq\left|h\left(b_{i v}^{\prime}\right)-h\left(a_{i v}\right)\right| / H^{2}
$$

for all $z \in \partial Q_{i}$ and for all $i, v$. Since $\left|b_{i v}^{\prime}-a_{i v}\right|=\left(\lambda_{i} / \mu\right)\left|b_{i v}-a_{i v}\right| \geqq\left|b_{i v}-a_{i v}\right| / 4$ and since $\left|a_{i v}-b_{i v}\right|=\left|b_{i v}^{\prime \prime}-b_{i v}\right| / 2$, (14) implies

$$
\left|h\left(b_{i v}^{\prime}\right)-h\left(a_{i v}\right)\right| \geqq \eta^{\prime}(1 / 4)\left|h\left(b_{i v}\right)-h\left(a_{i v}\right)\right| \geqq \eta^{\prime}(1 / 4) \eta^{\prime}(1 / 2) \beta_{i v}
$$

where $\beta_{i v}=\left|h\left(b_{i v}^{\prime \prime}\right)-h\left(b_{i v}\right)\right|$. It follows by (15) and (16) that for every $z \in \partial Q_{i v}$, $\left|h(z)-h\left(z_{i v}\right)\right| \geqq c \beta_{i v}$ where $c=\eta^{\prime}(1 / 2) \eta^{\prime}(1 / 4) / H^{2}=\mathrm{a}$ constant. Then $h\left(Q_{i v}\right) \supset$ int $B^{k+1}\left(h\left(z_{i v}\right), c \beta_{i v}\right)$ implying that the $(k+1)$-measure $m\left(h\left(Q_{i v}\right)\right)$ satisfies

$$
m\left(h\left(Q_{i v}\right)\right) \geqq c^{\prime} \beta_{i v}^{k+1}
$$

for a constant $c^{\prime}$.

Set now $\beta=\inf \left\{|h(x, 0, y)-h(x, 1, y)|: x \in A, y \in I^{k-1}\right\}>0$. Then for every $i$ and $v^{\prime}=\left(j_{2}, \ldots, j_{k}\right)$ we have $\beta \leqq \sum_{j=1}^{s} \beta_{i j v^{\prime}}$. Now the Hölder inequality and (17) imply that $\beta^{k+1} \leqq s^{k} \sum_{j} \beta_{i j v^{\prime}}^{k+1} \leqq s^{k} \sum_{j} m\left(h\left(Q_{i j v^{\prime}}\right)\right) / c^{\prime}$. Summing over $i$ and $v^{\prime}$, we get now

$$
r s^{k-1} \beta^{k+1} \leqq s^{k} \sum_{i, v} m\left(h\left(Q_{i v}\right)\right) / c^{\prime} \leqq s^{k} m\left(h\left(A \times I^{k}\right)\right) / c^{\prime} .
$$

Thus $r / s \leqq c^{\prime \prime}=$ a constant depending only on $h$ but not on $M$. On the other hand, $1 / s=\mu \geqq \lambda \geqq \lambda_{i}$. Thus $r / s \geqq \sum_{i} \lambda_{i} \geqq M$. This is a contradiction since we can choose $M>c^{\prime \prime}$.

Remark. In the above proof we have used only the following properties of the product metric (13): $\left|(x, y)-\left(x^{\prime}, y^{\prime}\right)\right| \geqq \min \left(\left|x-x^{\prime}\right|,\left|y-y^{\prime}\right|\right),\left|(x, y)-\left(x^{\prime}, y\right)\right|=$ $\left|x-x^{\prime}\right|$ and $\left|(x, y)-\left(x, y^{\prime}\right)\right|=\left|y-y^{\prime}\right|$. Since these are also true for several other natural product metrics, Lemma 4 is valid for such metrics, as well. 
Then we have, as first observed by S. Rickman (if $n=3$ ),

Theorem 5. The (n-1)-cell $S=J \times R^{n-2} \subset R^{n}$ is not quasiconformally locally flat.

Proof. If $x \in S$, if $U$ is a neighbourhood of $x$ in $R^{n}$ and if $h: U \rightarrow R^{n}$ is a quasiconformal embedding, then, by [14, 2.4], there is a smaller neighbourhood $V$ of $x$ such that $h \mid V$ is quasisymmetric. Since $n \geqq 3$, Lemma 4 implies that $h(V \cap S)$ cannot be contained in an $(n-1)$-plane of $R^{n}$. The theorem follows.

5. The examples. Before presenting the examples mentioned in the Introduction, we prove the following

Lemma 6. Let $m \geqq 1$ and $k \geqq 2$. Let $G$ be a Möbius group on $\bar{R}^{m}$ which is isomorphic to $R^{k}$ as a topological group. Then there is $a \in \bar{R}^{m}$ such that every $g \in G$ fixes $a$. If $\infty$ is fixed by every $g \in G$, there is a $k$-plane $V \subset R^{m}$ such that, restricted to $V, G$ acts as the group of translations of $V$.

Proof. If $g \in G$ is elliptic, then the subgroup generated by $g$ is contained in a compact group. Thus $\left\{g^{i}\right\}_{i>0}$ contains elements arbitrarily near the identity. We conclude that no $g \in G$ can be elliptic. Thus every $g \in G \backslash\{$ id $\}$ fixes exactly one or two points of $\bar{R}^{m}$. It is then easy to see that $g, g^{\prime} \in G \backslash\{$ id $\}$ can commute only if they have the same set of fixed points. We conclude that such a point $a \in \bar{R}^{m}$ exists, as claimed. If there is also another point $b$ fixed by every $g \in G$, then onc sees easily that either $G$ is isomorphic to $R$ or that $G$ contains elliptic elements. Both cases are impossible.

We have shown that every $g \in G \backslash\{\mathrm{id}\}$ fixes exactly one point, the point $a$, and hence $g$ is parabolic. We assume now that $a=\infty$. Then $R^{m}$ is $G$-invariant and we can regard $G$ as a group of $R^{m}$. If $g \in G \backslash\{\mathrm{id}\}, g$ is of the form

$$
g(x)=\beta\left(x-x_{0}\right)+a+x_{0},
$$

$x \in R^{m}$, for some $a, x_{0} \in R^{m}$ and for some orthogonal map $\beta$ of $R^{m}$ such that $\beta(a)=a$.

To conclude the proof of the lemma, we apply the Bieberbach theorems. Let $\Gamma \subset G$ be a discrete group such that $G / \Gamma$ is a $k$-torus, i.e. homeomorphic to $\left(S^{1}\right)^{k}$. Then the Bieberbach theorems imply that $\Gamma$ has a normal subgroup $\Gamma^{*}$ of finite index for which there is a $\Gamma^{*}$-invariant subplane $V \subset R^{m}$ such that $V / \Gamma^{*}$ is compact (Wolf [16, 3.2.8]). Choose $z \in V$. Since both $G z / \Gamma^{*}$ and $V / \Gamma^{*}$ are compact and $V \cap G z \supset \Gamma^{*} z$, there is $M>0$ such that

$$
d(x, V) \leqq M \quad \text { and } \quad d(y, G z) \leqq M
$$

for every $x \in G z$ and $y \in V$.

Let now $g \in \Gamma$ be arbitrary. Since $g(G z)=G z$, (19) implies that $g(V)$ must be parallel to $V$ and that $d(V, g(V)) \leqq 2 M$. If $V \neq g(V)$, then (18) implies that for some power $g^{k}$ of $g, d\left(V, g^{k}(V)\right)>2 M$. Thus $V$ is $\Gamma$-invariant. Since $V / \Gamma$ is compact, $[16,3.2 .9]$ implies that $g \mid V$ is a translation. Since obviously every $g \in G$ 
is contained in such a discrete subgroup $\Gamma$, we can conclude that $g \mid V$ is a translation for every $g \in G$. Now $G$ is isomorphic to $R^{k}$ and $V / \Gamma$ is compact. Consequently $V$ must be a $k$-plane such that $G$, restricted to $V$, is the group of translations of $V$.

In the following examples the groups $\bar{G}_{0}, G_{0}, \bar{G}_{1}, G_{1}$, the non-quasiconformal homeomorphism $F=F^{\prime} \times$ id of $K^{n}$, and the $(n-1)$-cell $S=J \times R^{n-2}$ are as in Section 3.

Example 1. Quasiconformal groups. Our first example answers the question posed by Gehring and Palka [6, p. 197].

The group $G_{0}$ is a Lipschitz group of $R^{n}$ such that for no quasiconformal homeomorphism $h$ of $R^{n}$ is the conjugation $h G_{0} h^{-1}$ a Möbius group (although $\bar{G}_{0}=F^{-1} G_{0} F$ is a group of translations of $R^{n}$ ).

The group $G_{1}$ is a quasiconformal group of $R^{n}$ which is not isomorphic as a topological group to any Möbius group on any $\bar{R}^{m}, m>0$ (although $\bar{G}_{1}=F^{-1} G_{1} F$ is a non-uniformly quasiconformal group of affine maps of $R^{n}$ ).

To see the validity of these claims, suppose first that $G^{\prime}=h G_{0} h^{-1}$ is a Möbius group, where $h$ is a homeomorphism of $R^{n}$. Since $G^{\prime}$ is clearly isomorphic as a topological group to $R^{n-1}$, Lemma 6 implies that the orbits $G^{\prime} z$ are $(n-1)$-planes of $R^{n}$. Thus, in particular, $h(S)$ is an $(n-1)$-plane. By Theorem $5, S$ is not quasiconformally locally flat and, hence, $h$ cannot be quasiconformal.

Next let $G$ be a Möbius group on some $\bar{R}^{m}$ and assume that there is an isomorphism of topological groups $\varphi: G_{1} \rightarrow G$. By Lemma 6 we can suppose that $\varphi\left(G_{0}\right)$ is a Möbius group of $R^{m}$. Lemma 6 also implies that there is an $(n-1)-$ plane $V \subset R^{m}$ such that $\varphi\left(G_{0}\right)$, restricted to $V$, is the group of translations of $V$.

Fix now $\lambda>0, \lambda \neq 1$ and let $g_{\lambda}$ be as in (8). Let $g=\varphi\left(g_{\lambda}\right)$. Since $G_{0}$ is a normal subgroup of $G_{1}, g h g^{-1} \in \varphi\left(G_{0}\right)$ for every $h \in \varphi\left(G_{0}\right)$. Hence $g$ must fix $\infty$. We let $h_{c}^{\prime} \in \varphi\left(G_{0}\right), c=u-v$ for some $u, v \in V$, be the map for which $h_{c}^{\prime} \mid V$ is the translation $x \mapsto x+c$. The map $g \mid R^{n}$ is a euclidean similarity of $R^{n}$. Thus, given $h_{c}^{\prime} \in \varphi\left(G_{0}\right)$, $g h_{c}^{\prime} g^{-1}=h_{c^{\prime}}^{\prime}$ with

$$
\left|c^{\prime}\right|=\lambda^{\prime}|c|
$$

for some $\lambda^{\prime}>0$ depending only on $g$ but not on $c$.

Now an isomorphism $\left(R^{k},+\right) \rightarrow\left(R^{k},+\right)$ of topological groups is necessarily a linear map. Then (20) combined with (9) gives a contradiction.

Example 2. Quasiconformal reflections. It is well-known that quasicircles in $\bar{R}^{2}$ can be characterized by the fact that they are topological circles which admit quasiconformal reflections. That is, if $C$ is such a circle, there is a quasiconformal map $r$ of $\bar{R}^{2}$ interchanging the components of $\bar{R}^{2 \backslash} \backslash C$ with $r \circ r=$ id and $r \mid C=\mathrm{id}$. We have by Theorem 5: 
The sphere $S \cup\{\infty\}$ admits a quasiconformal reflection but is not the image of the $(n-1)$-sphere $S^{n-1}$ under a quasiconformal map of $\bar{R}^{n}$ although it is of this form for a homeomorphism of $\bar{R}^{n}$.

Indeed, such a reflection can be obtained as a product $r \mid R^{n}=r_{0} \times \mathrm{id}, r(\infty)=\infty$, where $r_{0}$ is a quasiconformal reflection of $J$ in $R^{2}$ which is bilipschitz in the euclidean metric. There are always such reflections, cf. Ahlfors [1, p. 80]. Then $r \mid R^{n}$ is even bilipschitz in the euclidean metric which implies that $r$ is bilipschitz in the spherical metric. The last observation is due to J. Väisälä.

Example 3. Quasiconformal homogeneity. In $R^{2}$ one can characterize quasicircles by quasiconformal homogeneity $[2,3,4]$; in addition J. Sarvas (unpublished) has shown that if a Jordan domain $D \subset R^{2}$ is homogeneous with respect to a family of uniformly quasiconformal homeomorphisms of $R^{2}$, then $\partial D$ is a quasicircle. It would appear that in $R^{n}$ such characterizations fail since we have the following homogeneity properties for $S$ and its complementary domains:

The group $G_{0}$ is a Lipschitz group of $R^{n}$ acting transitively on the non-quasiconformally flat $(n-1)$-cell $S$.

The group $G_{1}$ is a quasiconformal group of $K^{n}$ acting transitively on $S$ and its complementary domains, which are homeomorphic to the open n-ball but not by a quasiconformal homeomorphism. In addition, restricted to a complementary domain of $S, G_{1}$ is a Lipschitz group in the quasihyperbolic metric.

These claims follow by Theorems 2 and 5 . We must only verify that, if $C$ is a comple mentary domain of $S$, then $C$ is not quasiconformally equivalent with the open $n$-ball $B$ of $R^{n}$, although it is clearly homeomorphic to it. Indeed, if $f$ were such a quasiconformal homeomorphism, we could first extend $f$ to $C \cup S \cup\{\infty\}$ and then, by the reflection of the preceding example, to the whole $\bar{R}^{n}$. Then Theorem 5 gives a contradiction.

Observe that the complementary domains of $S$ are uniform domains (cf. Martio-Sarvas [7, 2.12]) which follows easily from [7, 2.33] and the fact that $D \times R^{k} \subset R^{n}$ is uniform, whenever $D \subset R^{n-k}$ is unbounded and uniform. Thus the complementary domains of $S$ are also examples of uniform Jordan domains in $R^{n}$ not quasiconformally equivalent to the open $n$-ball. In fact, the question of the existence of such domains was the original motivation for the construction of $S$ by S. Rickman.

However, I do not know whether $\bar{S}=S \cup\{\infty\}$ is quasiconformally homogeneous. It is not Lipschitz homogeneous (in the spherical metric) since $\infty$ has arbitrarily small neighbourhoods $U$ such that every pair $x, y \in U$ can be joined by a rectifiable path in $U$. This is not true of other points of $\bar{S}$. In view of this fact I am inclined to think that $\bar{S}$ is not quasiconformally homogeneous. Nor do I know other examples of quasiconformally homogeneous spheres in $\bar{R}^{n}$ which are not quasispheres. This is due to the fact that in order to apply our method, $S^{n-1}$ would have to be the product of a 1-manifold and an $(n-1)$-manifold, which is not the case. 
To get an example of a Lipschitz homogeneous compact manifold in $\bar{R}^{3}$, we can take a torus which is homeomorphic to $S^{1} \times S^{1}$. Indeed, we can embed a torus $T$ in $\bar{R}^{3}$ in such a way that, although not locally quasiconformally flat, there is a Lipschitz group on $\bar{R}^{3}$ (in the spherical metric) which acts transitively on $T$. In this case, if $V$ is the $x z$-plane, $V \cap T$ consists of two nonrectifiable quasicircles $C_{1}$ and $C_{2}$, symmetric with respect to the $z$-axis. The torus $T$ is the trajectory of these circ'es as we turn $V$ round the $z$-axis. The quasicircles $C_{1}$ and $C_{2}$ are constructed like the arc $J$ in Section 3. Details are as above. Observe that the homeomorphism $F^{\prime}$ of $R^{2}$ such that $F^{\prime}(R)=J$ can be constructed in such a way that $F^{\prime}=$ id outside a suitable neighbourhood of $\bar{R}$ in $\bar{R}^{2}$ by the LIP annulus theorem, cf. e.g. [13, 3.4].

Now there is a homeomorphism $h$ of $\bar{R}^{3}$ such that if $G$ is the group described above, then $h G h^{-1}=G^{\prime} \times G^{\prime \prime}$ where $G^{\prime}$ is the group of rotations of $\bar{R}^{3}$ fixing the $z$-axis and $G^{\prime \prime}$ is the group of rotations fixing $S^{1}$. It is a group of isometries of $\bar{R}^{3}$ in the spherical metric.

Observe that now $G$ is isomorphic to $S^{1} \times S^{1}$ as a topological group. Thus it contains a one-generator subgroup $\bar{G}$ which is dense in $G$. Then $\bar{G}$ is an examp'e of a one-generator Lipschitz group which cannot be conjugated by a quasiconformal map into a Möbius group.

For a discussion of quasiconformally homogeneous domains of $R^{n}$ see [6].

Example 4. Quasisymmetric manifolds. A quasisymmetric manifold is a metric space which is an $n$-manifold such that every $x \in M$ has a neighbourhood which can be embedded in $R^{n}$ by a quasisymmetric mapping. Since the map $F^{\prime-1} \mid J: J \rightarrow R$ is quasisymmetric by $[14,2.4]$, we have by Theorem 5 :

There are quasisymmetric manifolds $M_{1}(=J)$ and $M_{2}\left(=R^{n-2}\right)$ such that $M_{1} \times M_{2}(=S)$ is not a quasisymmetric manifold in the product metric given by (13).

We remark that this remains true even if one replaces our definition of a quasisymmetric mapping by the stronger definition of $[12,1.1]$. This definition is in the present context more appropriate since one now gets immediately an atlas whose maps are quasisymmetric. This is not clear if one uses the definition in the present paper.

Example 5. Quasiconformal spheres. The $(n-1)$-cell $S$ is also a counterexample to Conjecture 3 in my paper [9, p. 71] where I attempted to characterize quasiconformal $k$-spheres in $\bar{R}^{n}$ by means of a condition resembling the characterization of quasiconformal homeomorphisms of $R^{n}$ by means of compact families of mappings, ([5, Theorem 18]). This characterization generalizes to quasisymmetric embeddings [12, 3.21]. In the present conjecture compact families of mappings are replaced by compact families whose elements are subsets of $R^{n}$. However, it is easy to see that if in Conjecture $3 C=S$, then every set $X \in \operatorname{cl} \mathscr{F}_{c}$ is of the form $g\left(R^{n}\right)$ for some homeomorphism of $R^{n}$, thus disproving the conjecture. 


\section{References}

[1] Ahlfors, L. V.: Lectures on quasiconformal mappings. - D. Van Nostrand Company Inc.,, Princeton, New Jersey-Toronto-New York-London, 1966.

[2] Blevins, D. K., and B. P. Palka: A characterization of quasicircles. - Proc. Amer. Math. Soc. 50, 1975, 328-331.

[3] Brechner, B., and T. Erkama: On topologically and quasiconformally homogeneous continua. - Ann. Acad. Sci. Fenn. Ser. A I Math. 4, 1978/1979, 207-208.

[4] Erkama, T.: Quasiconformally homogeneous curves. - Michigan Math. J. 24, 1977, 157-159.

[5] Gehring, F. W.: Rings and quasiconformal mappings in space. - Trans. Amer. Math. Soc. 103, 1962, 353-393.

[6] Gehring, F. W., and B. P. Palka: Quasiconformally homogeneous domains. - J. Analyse Math. 30, 1976, 172-199.

[7] Martio, O., and J. Sarvas: Injectivity theorems in plane and space. - Ann. Acad. Sci. Fenn. Ser. A I Math. 4, 1978/1979, 383-401.

[8] Sullivan, D.: On the ergodic theory at infinity of an arbitrary discrete group of hyperbolic motions. - Riemann surfaces and related topics: poceedings of the 1978 Stony Brook conference. edited by I.Kra and B.Maskit, Annals of Mathematics Studies 97, Princeton University Press, Princeton, New Jersey, 1981, 465-496.

[9] Tukia, P.: The planar Schönflies theorem for Lipschitz maps. - Ann. Acad. Sci. Fenn. Ser. A I Math. 5, 1980, 49-72.

[10] Tukia, P.: On two-dimensional quasiconformal groups. - Ibid. 5, 1980, 73-78.

[11] TUKIA, P.: Extension of quasisymmetric and Lipschitz embeddings of the real line into the plane. - Ibid. 6, 1981, 89-94.

[12] TukiA, P., and J. VÄIsÄLÄ: Quasisymmetric embeddings of metric spaces. - Ibid. 5, 1980, 97-114.

[13] VÄISÄLÄ, J.: Piecewise linear approximation of lipeomorphisms. - Ibid. 3, 1977, 377-383.

[14] VÄIs̈̈LÄ, J.: Quasisymmetric embeddings in euclidean spaces. - Trans. Amer. Math. Soc. 264, 1981, 191-204.

[15] VÄIsÄL $\ddot{A}$, J.: Lectures on $n$-dimensional quasiconformal mappings. - Lecture Notes in Mathematics 229, Springer-Verlag, Berlin-Heidelberg-New York, 1971.

[16] Wolf, J. A.: Spaces of constant curvature. - Publish or Perish, Inc.. Berkeley, 1977.

University of Helsinki

Department of Mathematics

SF-00100 Helsinki 10

Finland

Received 24 November 1980 